\title{
Waveguide-coupled round-corner octagonal microresonator channel add-drop filters
}

\author{
Chao Li and Andrew W. Poon \\ Department of Electrical and Electronic Engineering, The Hong Kong University of Science and Technology, \\ Clear Water Bay, Hong Kong SAR, China \\ Tel: (852)-2358-7905, fax: (852)-2358-1485, email: eeawpoon@ust.hk
}

\begin{abstract}
We report the first waveguide-coupled round-corner octagonal microresonator channel add-drop filters on silicon-nitride-on-silica substrates. By using photolithography and plasma etching, we fabricated a waveguide-coupled 50-micron sized octagonal microresonator with 15-micron radius round corners. Our experiment reveals TM-polarized whispering-gallery-like modes with a $\mathrm{Q}$ of about 3800 and a finesse of 18.

(C) 2004 Optical Society of America

OCIS codes: (230.5750) Resonators; (230.3990) Microstructure devices
\end{abstract}

High-index contrast microresonators ( $\mu$-resonators) have been gaining considerable interest for wavelength-division multiplexing channel add-drop applications $[1,2]$. Recently, laterally waveguide-coupled octagonal $\mu$-resonator filters have been demonstrated on silicon-nitride-on-silica substrates [1]. Octagonal $\mu$-resonators have the advantage of flat cavity sidewalls for lateral coupling with straight waveguides. The interaction length along the entire flat resonator sidewall can ease the stringent fabrication constraint imposed by the sub-micrometer air-gap separation for lateral coupling [1]. However, octagonal $\mu$-resonators have excess cavity loss due to the sharp cavity corners [1]. In this abstract, we report our initial experimental results on a modified octagonal $\mu$-resonator filter with rounded cavity corners in order to mitigate the cavity corner loss.

We employed standard silicon microelectronic fabrication processes similar to Ref. [1]. A silica under-cladding layer of about $1.5 \mu \mathrm{m}$ thick was deposited by wet oxidation on a silicon substrate, followed with the low-pressure chemical vapor deposition of a low-stress silicon nitride film of about $1.1 \mu \mathrm{m}$ thick. The stack-layered structure was air-clad. The filter pattern was defined by photolithography and transferred onto the nitride layer by plasma etching. The devices have an etched depth of about $0.95 \mu \mathrm{m}$.

(a)

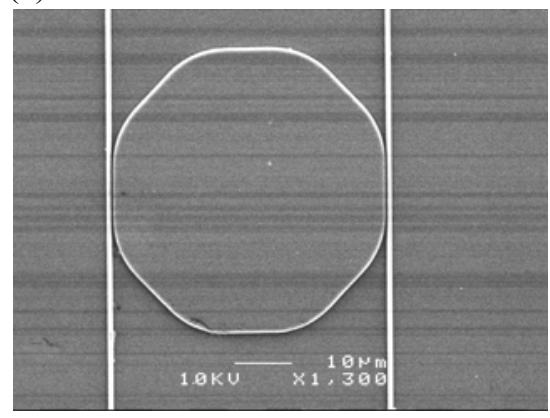

(b)

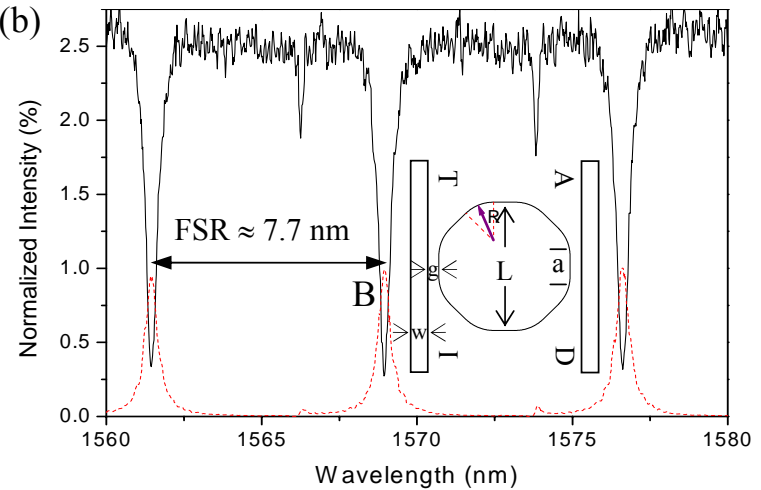

Fig. 1. (a) Electron micrograph of a waveguide-coupled round-corner octagonal $\mu$-resonator channel add-drop filter. (b) Measured TM-polarized throughput (solid) and drop (dashed) spectra of the filter. Inset shows the design schematic. I: input, T: throughput, D: drop, A: add. $\mathrm{L}=50 \mu \mathrm{m}, \mathrm{R}=15 \mu \mathrm{m}, \mathrm{a}=8.3 \mu \mathrm{m}, \mathrm{w}=0.6 \mu \mathrm{m}$ and $\mathrm{g}=0.35 \mu \mathrm{m}$.

Fig. 1 (a) shows the electron micrograph of a fabricated waveguide-coupled octagonal $\mu$-resonator filter. Fig. 1 (b) shows the preliminary measured TM-polarized (E-field $\perp$ plane) spectra of the filter. Inset shows the schematic design. An arc of $45^{\circ}$ with a radius $\mathrm{R} \approx 15 \mu \mathrm{m}$ is applied to each cavity corner. Mode $\mathrm{B}$ is preferentially coupled with about $90 \%$ efficiency, a Q of about 3,800, and a finesse of 18 . The free spectral range (FSR) is about $7.7 \mathrm{~nm}$, which is consistent with a round-trip path length slightly less than the cavity circumference. We refer to this mode as a whispering-gallery-like mode. Further experimental and numerical investigations are in progress in order to optimize the round-corner octagonal $\mu$-resonator filter design.

\section{References}

[1] C. Li, N. Ma, and A. W. Poon, "Waveguide-coupled octagonal microdisk channel add-drop filters," Opt. Lett., 29, pp. 471-473, 2004.

[2] T. Barwicz, M. A. Popović, P. T. Rakich, M. R. Watts, H. A. Haus, E. P. Ippen, and H. I. Smith, "Microring-resonator-based add-drop filters in SiN: fabrication and analysis," Opt. Express, 12, pp. 1437-1442, 2004. 